\title{
Inter-genotype variation in reproductive response to crowding among Daphnia pulex
}

\author{
Jay M. Fitzsimmons ${ }^{1}$ \& David J. Innes ${ }^{2, *}$ \\ ${ }^{1}$ Department of Biological Sciences, University of Windsor, N9B 3P4, Windsor, Ontario, Canada \\ ${ }^{2}$ Department of Biology, Memorial University of Newfoundland, AlB 3X9, St. John's, Newfoundland and Labrador, \\ Canada \\ (*Author for correspondence: E-mail: dinnes@mun.ca)
}

Received 21 September 2005; in revised form 10 February 2006; accepted 15 February 2006; published online 5 May 2006

Key words: Daphnia pulex, crowding stress, reproduction, offspring sex ratio, inter-genotype variation, maternal age

\begin{abstract}
Crowding is known to have a major influence on reproduction in the freshwater microcrustacean Daphnia pulex. We analyzed reproductive output of six different $D$. pulex genotypes under two different density regimes in the laboratory. Four of these genotypes reproduce via obligate parthenogenesis, allowing thorough analysis of the life history strategies of some asexual lines. Among 30,109 neonate offspring and 1041 resting egg ephippia collected, several trends were evident. Crowding induced increased resting egg production and reduced neonate offspring production among all genotypes. Offspring sex ratios grew more male-biased with maternal age. The extent, but not direction, of each of these trends varied among genotypes. Offspring sex ratios, and the very direction in which they changed in response to crowding, differed significantly among genotypes with some genotypes producing more and others fewer males in response to crowding. Obligately parthenogenetic genotypes seemed to respond to the crowding stimulus in similar ways as the facultatively parthenogenetic genotypes, as expected from the sexual origins of their genomes. The inter-genotype variation in life-history traits observed in this and other investigations calls into question the common practice of extrapolating results from a single Daphnia genotype to an entire species. Our findings are considered in the context of other research in the field of environmental influences on Daphnia reproduction with a review of representative literature.
\end{abstract}

\section{Introduction}

Reproduction does not necessarily follow the simple mantra of more is better. There are tradeoffs. Investing too much in reproduction early in life may leave an organism with fewer resources available for defence, growth, and future reproductive investment. The amount and type of reproductive output must also match the environment in which the offspring will grow. Thus the utilization of appropriate predictive cues can be just as important as reproductive investment itself.
The freshwater zooplankton Daphnia pulex (Leydig) (Crustacea: Cladocera) is well-suited to experimental research on life-history strategies. Like other model organisms, it is easily reared in the laboratory, has short generation times, and the basic components of its ecology and life-histories are known from previous research. It may reproduce via clonal free-swimming offspring (neonate) production or diapausing resting egg production (in ephippia), so trade-offs between short- and long-term reproductive investment can be observed. Clonal offspring production allows comparison of essentially the same genome across 
several environmental conditions, which is a major advantage of using $D$. pulex in life-history research.

Crowding is known to affect reproductive type (neonate vs. resting egg) and amount among Daphnia. Crowding may precede one of several potential environments. Ephemeral populations of Daphnia tend to follow boom-and-bust cycles, whereby high densities often precede steep declines (Dudycha, 2004; Nelson et al., 2005). Crowding may also indicate decreasing water levels as a result of the pond drying up. Either of these situations should favour investment in diapause as opposed to neonate production. Accordingly, Daphnia have been found to produce more ephippia and fewer neonates in response to crowding (Berg et al., 2001; Lürling et al., 2003).

Crowding may also predict offspring environment to be more crowded and competitive, with potentially greater risk of horizontal transmission of disease and parasites. In this situation, whatever neonate offspring are produced should be of overall high quality, being resistant to food stress and parasites. Investment in higher-quality offspring may necessitate a concomitant decrease in offspring quantity. Research suggests crowded Daphnia mothers respond to crowding in similar ways as to food stress (Burns, 1995). Crowded mothers produce fewer neonates than uncrowded mothers, but these offspring tend to be larger (Gliwicz \& Guisande, 1992; Cleuvers et al., 1997), develop more rapidly (Cleuvers et al., 1997), and be more resistant to starvation (Gliwicz \& Guisande, 1992) and parasites (Mitchell \& Read, 2005). Whether the production of high-quality neonates is a response to crowding in specific or to stresses in general remains to be determined. Daphnia magna Straus have been found to produce offspring with greater resistance to toxins, for instance, both when the mother is exposed to crowding (Baird et al., 1991) and to toxins (Gustafsson et al., 2005).

Several studies have examined the effect of crowding on various aspects of $D$. pulex reproduction (e.g., Ruvinsky et al., 1986; Innes et al., 2000; Berg et al., 2001). Our research explored the effects of crowding on $D$. pulex reproduction in terms of amount, mode (neonate vs. ephippia), and neonate sex ratio. Unlike some other research, we utilized six different genotypes (or clones, henceforth used interchangeably) in order to compare genotypes' strategies and their responses to crowding (genotype $\times$ environment interactions). Genotype $\times$ environment interactions are known to have a great effect on Daphnia fitness and life-histories (Mitchell et al., 2005), as they likely do for most species. We also observed reproductive output in relation to maternal age, allowing age-specific strategies to be revealed.

\section{Life cycle of Daphnia pulex}

The life cycle of $D$. pulex must be considered in the context of its habitat; ephemeral freshwater ponds. It is only the resting egg stage of the D. pulex life cycle that can survive over winter or when the pond has dried up. Daphnia resting eggs may hatch the following year, or may remain buried and hatch many years later (Kerfoot \& Weider, 2004). Normally two resting eggs are encased within an ephippium, which is a hard dark case that protects the eggs from stresses.

Daphnia pulex individuals can reproduce either by facultative parthenogenesis (FP) or obligate parthenogenesis (OP). Facultative parthenogenesis is also referred to as cyclical parthenogenesis in much of the literature, but a change in terminology to FP has been suggested (Tessier \& Cáceres, 2004). Both FP and OP D. pulex readily produce neonates clonally, but FP lines require sex and recombination to produce resting eggs, while OP line females produce resting eggs asexually with resulting genotypes matching those that would be expected from mitosis (Hebert, 1987). Method of reproduction is believed to be inherited genetically (Innes \& Hebert, 1988). Although many northern OP lines are polyploid (Hebert, 1987), allozyme electrophoresis results (not shown) led us to believe the genotypes used here were diploid.

Some OP lines are capable of producing males (Hebert et al., 1989; Innes et al., 2000). These males are functional, and can mate with FP females to produce a mixture of FP and OP offspring (Innes \& Hebert, 1988). Although a clonal line may be obligately parthenogenetic, it will retain much of the genetic history of sexual $D$. pulex (Lynch et al., 1989). Cues to induce male production, for instance, may no longer be relevant to OP lines, but with genomes that are essentially 
frozen OP lines may remain responsive to these cues.

Neonate sex is determined environmentally with genetic contributions (Innes \& Singleton, 2000). Thus both male and female offspring are genetically identical. Neonates are produced in broods that range in size from several to dozens. All neonates within a brood are usually the same sex, although mixed-sex broods are not uncommon (Innes, 1997). Male and female neonates resemble small versions of their adult equivalents, and can be distinguished morphologically based on the presence of a distinct second antennule among males.

A key component of $D$. pulex fitness is a genotype's ability to produce an optimal number of offspring via neonate production to maximize the number of resting eggs produced before pond conditions deteriorate. The cues used by $D$. pulex to induce male- and resting-egg production vary across genotypes within and between habitats, and include crowding (Berg et al., 2001).

\section{Materials and methods}

\section{Clonal isolates}

Specimens were sampled from four ephemeral ponds in the Great Lakes watershed of North America in May 2003, using previously described methods (Innes, 1997). Disp and VBA ponds are in Windsor and Guelph, Ontario, respectively (Fitzsimmons \& Innes, 2005). Morg and War are near Ann Arbor, Michigan, being previously described as ponds 69 and 73 respectively (Hebert \& Crease, 1983). All ponds are similar in size and are believed to support $D$. pulex populations in the spring of each year only. Disp and Morg occur in woodlots, while VBA and War ponds are in more open areas. At least a dozen clonal isolates were individually maintained from each of these ponds in synthetic zooplankton media (Lynch et al., 1986) in conditions described elsewhere (Innes \& Dunbrack, 1993). Cups of clonal strains were fed $3.5 \mathrm{~mL}$ of algal slurry from an aquarium system daily. The goldfish in the aquarium were fed fish food flakes, and their excretions provided nutrients for a small variety of phytoplankton and bacteria to thrive. Though the small random fluctuations in this algal community prevent precise quantifications, its multi-taxa composition is a more natural food than single algal strains, and may increase Daphnia reproductive output (Sanders et al., 1996).

The clones chosen for the experiment had been observed with males on multiple occasions in the lab. This non-random sampling of genotypes was purposeful, as only genotypes capable of producing males were desired. One clone from each of Disp and VBA was chosen, as were two clones from each of Morg and War. The two clones from Morg and War were genetically unique, as inferred from allozyme electrophoresis. It was originally believed that all six of these clones were OP, based on previous research with these populations (Hebert et al., 1989) and phenotypic heterozygosity at the lactate dehydrogenase (LDH) allozyme locus, which is usually correlated with obligate parthenogenesis (D. J. Innes, personal observation). Verification of mode of reproduction, however, strongly suggested the clones Disp and War2 were FP. Verification entailed allowing females to produce ephippia in the absence of males. OP daphnids can produce diapausing resting eggs within ephippia in the absence of males, whereas FP daphnids can only produce an empty ephippium (Innes et al., 1986). Since OP daphnids may also occasionally produce empty ephippia, however, a single empty ephippium is insufficient evidence for facultative parthenogenesis. More than 20 ephippia were opened for each of Disp and War2 without the presence of resting eggs, while ephippia containing resting eggs were found for each of the other clones within the first 3 being opened. We therefore assume Disp and War2 to be FP.

Additional FP and OP genotypes were included in the experiment initially, and would have allowed more general tests of FP vs. OP reproductive investment. However the amount of time required for accurate counting and sexing neonates necessitated a reduction in the number of genotypes tested. We do not feel it prudent to test for general differences between FP and OP D. pulex reproductive investment from such a small sample.

Three successive parthenogenetic generations of each clone, originating from a single female of each clonal culture, were raised in identical conditions in an incubator prior to the experiment. This was done to control for any potential 
maternal or grandmaternal effects and to prevent mortality of clones unaccustomed to lab conditions (Antunes et al., 2003). These conditions were $15^{\circ} \mathrm{C}, 16 \mathrm{~L}: 8 \mathrm{D}$ photoperiod, $3.5 \mathrm{~mL}$ of algae daily, and 5 females per $80 \mathrm{~mL}$ cup of media. Female neonates were obtained from the final generation within $24 \mathrm{~h}$ of being released from a brood and used in the experiment.

\section{Experimental set-up}

Translucent $140 \mathrm{~mL}$ plastic cups were filled with $40 \mathrm{~mL}$ of synthetic zooplankton media. Either 1 or 10 female neonates of a clone were included in a cup, for Alone and Crowded conditions, respectively. It should be noted that even the Alone condition may be considered somewhat crowded to Daphnia (Banta \& Brown, 1929; Hobæk \& Larsson, 1990). Eight replicate cups were used for each clone in each density condition (2 density conditions $\times 6$ clones $\times 8$ replicates $=96$ cups $)$. Cups were maintained in incubators at $15^{\circ} \mathrm{C}$, with a short-day photoperiod of $8 \mathrm{~L}: 16 \mathrm{D}$. Alone and Crowded cups were fed 1.9 and $3.8 \mathrm{~mL}$ of algae daily respectively. Crowded cups were thus fed $2 \times$ as much algae, but had $10 \times$ more daphnids, and the results of our experiment may be either due to differences in density or food levels. We note that stock cultures of several dozen reproducing daphnids were maintained on only $2 \mathrm{~mL}$ of algae daily, suggesting food levels were ample in both treatment conditions.

Every other day the daphnids were transferred by pipette to a cup of new media, and the contents of the old media poured through a $250 \mu \mathrm{m}$ plankton mesh to collect all neonates and ephippia. Neonates were temporarily immobilized in a shallow, weak ethanol-water solution to allow easier visualization and counting under a dissecting microscope. Neonate sex was determined based on visualization under the microscope. The numbers of male and female neonates were recorded along with number of ephippia. Ephippia were not opened to count the number of resting eggs within. After data collection, neonates and ephippia were discarded.

Data collection continued until the specimens reached the age of 38 days, at which point the experiment was terminated. Although D. pulex individuals can live longer than 1 month in a lab environment, their lifespan is likely shorter than 1 month in their natural habitat (Dudycha, 2004), so it made little sense to extend the experiment beyond 38 days of age. Spare replicates were kept for most clones, under identical conditions as others, to replace dead experimental daphnids. No daphnids in the Alone condition died in the experiment, but a small number died in the Crowded condition. Most of these were immediately replaced with individuals of the same genotype, age, condition, and stage of reproduction (i.e., carrying ephippia, carrying early-stage brood, etc.). This resulted in balanced statistical analyses.

\section{Data analyses}

All data were analyzed using MINITAB 14.1, and all graphs were produced using Prism 4.0. The General Linear Model (GLM) was the primary method used to evaluate each of the four major aspects of the data: Ephippia production, experiment-long fecundity, brood size, and sex ratio.

To test the various factors influencing variation in ephippia production, the factors of Density and Clone were included along with their interaction. The response variable was the total ephippia production over the length of the experiment per adult female in experimental cup. If ephippia production were no different between Alone and Crowded conditions, then per-female ephippia production would not differ between density conditions. Ephippia data were consolidated over the length of the experiment as opposed to analyzed at individual data points for two reasons: non-consolidated data produced residuals that violated all assumptions required for GLM, and Age was determined to not be a significant factor influencing ephippia production in these analyses (results not shown).

Total fecundity over the length of the experiment was calculated using neonate data only, since any incorporation of ephippia in fecundity would necessitate a weighting factor to be applied to it, which may or may not be accurate. With total neonate production per female over the length of the experiment as the response variable, terms in the model were Density, Clone and Density $\times$ Clone interaction.

The assumption is made in many Daphnia studies that male and female neonates cost the 
same amount of resources to the mother (Korpelainen, 1992; Innes, 1997). This assumption is based largely on analyses by Barker \& Hebert (1986) in which separate mixed model ANOVAs were performed on each of four clones of D. magna, testing for the influences of brood sex and date of brood release (i.e., age of mother) on brood size within each clone. In each of these analyses the date of brood release, but not brood sex, had a significant effect on brood size (Barker $\&$ Hebert, 1986). Since the sex of a brood had no significant effect on the number of neonates in the brood, males and females were assumed to cost the same resources for the mother. Similar withinclone comparisons of male and female brood sizes have been conducted on a single clone of D. magna (Hobæk \& Larsson, 1990) and three clones of D. pulex (Innes \& Singleton, 2000).

We tested our data in two ways, using a separate mixed model ANOVA for each relevant clone with the terms age of mother at brood release (Age, regression term) and brood sex (BrdSex) as was done by Barker \& Hebert (1986), as well as using the GLM outlined below. Brood size could only be evaluated from daphnids in the Alone density condition, since separate broods could not be distinguished in the Crowded condition. The few mixedsex broods observed in the Alone condition were excluded from analysis, so as not to confound the BrdSex factor. Because Morg2 and VBA did not produce both all-male and all-female broods in the Alone condition, all data for these clones were excluded from brood size analyses evaluating the effect of brood sex. We believe the GLM analysis evaluates the assumption of equal resource costs for male and female neonates in a more robust manner than clone-specific ANOVAs. The GLM incorporates all relevant clones' data into one model, and adds the Clone factor to determine the level of variation in brood size that can be explained by variation among clones. If our analyses were to find brood sex to significantly influence brood size, then assumptions of equal resource cost for male and female neonates would require re-evaluation.

The GLM to evaluate factors influencing brood size had the number of neonates in a brood (BrdSize) as its response variable. The terms in the model were: Age, Clone, BrdSex, and the two-way interactions Age $\times$ Clone, Age $\times$ BrdSex, and Clone $\times$ BrdSex. This GLM was evaluated using the same four clones mentioned above. If all factors involving BrdSex were found to be insignificant, then a new GLM would be created that would eliminate such terms and only include the factors Age, Clone, and their interaction. This GLM would include data from all six clones, since there would be no reason to exclude Morg2 and VBA for producing broods of one sex only.

Although sex ratio is a common subject of study, its analysis is difficult with Daphnia. Because all neonates within a $D$. pulex brood tend to be the same sex, assumptions of sex independence are violated for within-brood siblings. For this reason, a common way to analyze sex ratios or sex allocation in Daphnia research has been to classify each brood as either all-female, or containing any males (e.g., Hobæk \& Larsson, 1990; Olmstead \& LeBlanc, 2002). Because broods could not be distinguished from each other in the Crowded condition of our data, this method would not suffice for our purposes. Thus we totalled all neonate data across the length of the experiment for each replicate, producing lifetime sex ratios (total males over total neonates produced). This Sex Ratio was the response variable for our analysis, and the terms in the GLM were Density, Clone, and Density $\times$ Clone interaction.

Using consolidated data for sex ratios prevented the evaluation of Age as a factor influencing sex ratio variation. To evaluate its influence, another GLM was analyzed using only Crowded condition data, not consolidated over the length of the experiment. Data from the clone VBA were excluded since no males were produced in the Crowded condition. With Sex Ratio as the response variable, the terms of the model were Age (regression), Clone, and Age $\times$ Clone interaction.

To evaluate whether any effect of Age on sex ratios was due to an extraordinary bias of brood sex among first broods of $D$. pulex, we also compared sex ratio data between sequential broods. Since separate broods could not be distinguished in the Crowded condition, only data from the Alone condition were used. Data from Morg2 and VBA were excluded since neither produced both male and female broods in the Alone condition. Each replicate's broods were distinguished by the order in which they occurred. $t$-Tests of the brood data were carried out comparing the sex ratios of 
first broods with those of second broods, and between second and third broods.

\section{Results}

A total of 30,109 neonates and 1041 ephippia were collected during the experiment (Table 1). Assumptions of residuals' independence, homogeneity, and sums of zero are made in any analyses based on General Linear Models (GLMs), and are met for all analyses here.

\section{Ephippia production}

Of the 1041 ephippia collected in this experiment, only $20(1.92 \%)$ came from daphnids in the Alone condition, all of which were from the Morg2 clone (Table 1). This is reflected in the statistical results. The Density $\times$ Clone interaction explained a significant amount of the variation in experiment-long ephippia production per adult female $(p<0.001$, Table 2$)$. The individual terms Density and Clone also explained significant amounts of variation $(p<0.001$ for each, Table 2), but this must be interpreted with caution since the interaction term is significant and takes precedence. The extent of the increase in number of ephippia produced from Alone to Crowded conditions depended upon the clone (Fig. 1).

\section{Fecundity}

The total number of neonates produced by each daphnid varied according to both clone and density condition, with crowded individuals of each clone producing fewer neonates per-daphnid than those in the Alone condition (Table 1, Fig. 2). The interaction Density $\times$ Clone explained a significant amount of the variation in neonate production $(p<0.001$, Table 2). The individual terms Density

Table 1. Numbers of neonates and ephippia collected in the experiment

\begin{tabular}{|c|c|c|c|c|c|c|}
\hline Clone & Density & Male neonates & Female neonates & Total neonates & Sex ratio ( $\%$ males $)$ & Ephippia \\
\hline \multirow[t]{3}{*}{ Disp (FP) } & Alone & 453 & 114 & 567 & 79.89 & 0 \\
\hline & Crowded & 2925 & 585 & 3510 & 83.33 & 42 \\
\hline & Total & 3378 & 699 & 4077 & 82.86 & 42 \\
\hline \multirow[t]{3}{*}{ Morg1 (OP) } & Alone & 71 & 1154 & 1225 & 5.80 & 0 \\
\hline & Crowded & 730 & 2163 & 2893 & 25.23 & 179 \\
\hline & Total & 801 & 3317 & 4118 & 19.45 & 179 \\
\hline \multirow[t]{3}{*}{ Morg2 (OP) } & Alone & 10 & 463 & 473 & 2.11 & 20 \\
\hline & Crowded & 42 & 332 & 374 & 11.23 & 345 \\
\hline & Total & 52 & 795 & 847 & 6.14 & 365 \\
\hline \multirow[t]{3}{*}{ VBA (OP) } & Alone & 2 & 1558 & 1560 & 0.13 & 0 \\
\hline & Crowded & 0 & 6599 & 6599 & 0.00 & 95 \\
\hline & Total & 2 & 8157 & 8159 & 0.02 & 95 \\
\hline \multirow[t]{3}{*}{ War1 (OP) } & Alone & 553 & 1194 & 1747 & 31.65 & 0 \\
\hline & Crowded & 3060 & 4897 & 7957 & 38.46 & 70 \\
\hline & Total & 3613 & 6091 & 9704 & 37.23 & 70 \\
\hline \multirow[t]{3}{*}{ War2 (FP) } & Alone & 1589 & 101 & 1690 & 94.02 & 0 \\
\hline & Crowded & 919 & 595 & 1514 & 60.70 & 290 \\
\hline & Total & 2508 & 696 & 3204 & 78.28 & 290 \\
\hline \multirow[t]{3}{*}{ Overall } & Alone & 2678 & 4584 & 7262 & 36.88 & 20 \\
\hline & Crowded & 7676 & 15,171 & 22,847 & 33.60 & 1021 \\
\hline & Total & 10,354 & 19,755 & 30,109 & 34.39 & 1041 \\
\hline
\end{tabular}

Clonal method of resting egg production is designated as facultative parthenogenetic (FP) or obligately parthenogenetic (OP). Daphnids were kept at densities of one (Alone) or ten (Crowded) in $40 \mathrm{~mL}$ of liquid. 
Table 2. General Linear Model results for several analyses

\begin{tabular}{|c|c|c|c|c|c|c|}
\hline & Factor & d.f. & Seq SS & Adj MS & $F$ & $p$ \\
\hline \multirow[t]{5}{*}{ Ephippia } & Density & 1 & 74.016 & 74.016 & 248.13 & $<0.001$ \\
\hline & Clone & 5 & 119.306 & 23.861 & 79.99 & $<0.001$ \\
\hline & Density $\times$ Clone & 5 & 27.302 & 5.460 & 18.31 & $<0.001$ \\
\hline & Error & 84 & 25.056 & 0.298 & & \\
\hline & Total & 95 & 245.681 & & & \\
\hline \multirow[t]{5}{*}{ Fecundity } & Density & 1 & 256739 & 256739 & 294.87 & $<0.001$ \\
\hline & Clone & 5 & 187254 & 37451 & 43.01 & $<0.001$ \\
\hline & Density $\times$ Clone & 5 & 65517 & 13103 & 15.05 & $<0.001$ \\
\hline & Error & 84 & 73136 & 871 & & \\
\hline & Total & 95 & 582647 & & & \\
\hline \multirow[t]{5}{*}{ Brood Size } & Age & 1 & 23718.4 & 19895.4 & 366.16 & $<0.001$ \\
\hline & Clone & 5 & 14356.4 & 543.2 & 10.00 & $<0.001$ \\
\hline & Age $\times$ Clone & 5 & 8354.6 & 1670.9 & 30.75 & $<0.001$ \\
\hline & Error & 248 & 13475.3 & 54.3 & & \\
\hline & Total & 259 & 59904.7 & & & \\
\hline \multirow[t]{5}{*}{ Sex Ratio } & Density & 1 & 0.03109 & 0.01089 & 0.64 & 0.427 \\
\hline & Clone & 5 & 9.33902 & 1.87192 & 109.60 & $<0.001$ \\
\hline & Density $\times$ Clone & 5 & 0.99187 & 0.19837 & 11.61 & $<0.001$ \\
\hline & Error & 82 & 1.40054 & 0.01708 & & \\
\hline & Total & 93 & 11.76251 & & & \\
\hline
\end{tabular}

See Methods (Data Analyses) for details on each analysis and factor.

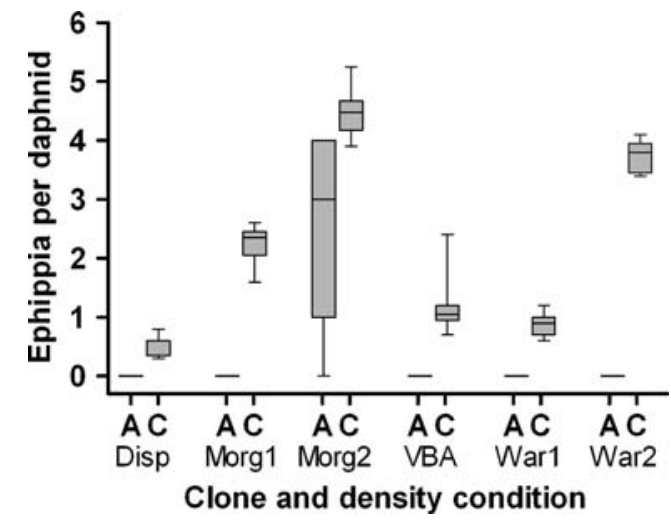

Figure 1. Per-daphnid ephippia production among clones in the Alone (A) and Crowded (C) conditions. Boxes include the median and span the 25th-75th percentiles. Whiskers span the range of the data.

and Clone also explained significant amounts of this variation $(p<0.001$ for each, Table 2$)$. All GLM-based assumptions of residuals were met. As would be expected, there was a significant negative correlation among Crowded condition daphnids

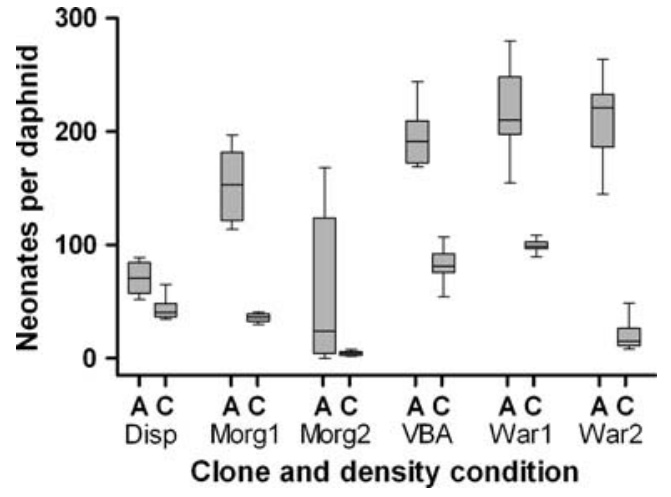

Figure 2. Experiment-long fecundity per daphnid among clones in the Alone (A) and Crowded (C) conditions. Boxes include the median and span the 25 th-75th percentiles. Whiskers span the range of the data.

between the numbers of neonates and ephippia produced over the length of the experiment (Pearson correlation $r=-0.799, p<0.001$, Fig. 3). A daphnid can carry either a brood of neonates or an ephippium at one time, resulting in a trade-off. 


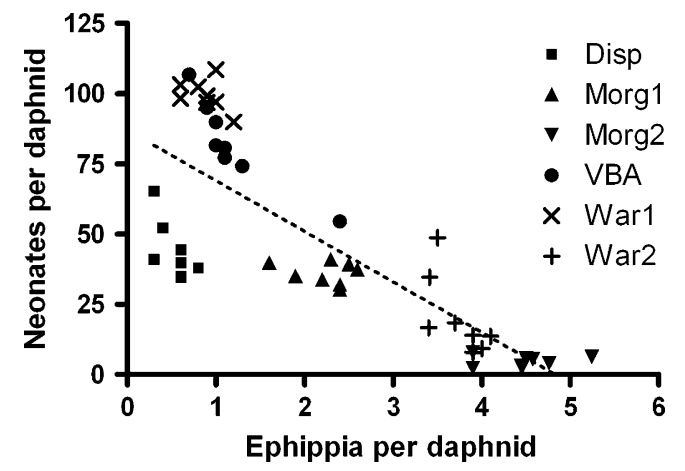

Figure 3. Experiment-long production of neonates and ephippia per daphnid among clones in the Crowded condition. The six different clones are designated by points of different shapes, with eight replicates each. The black dotted line represents the linear regression among all data points.

\section{Brood size}

Like Barker \& Hebert (1986), we tested for the influence of brood sex and age on brood size in separate ANOVAs for each of the four appropriate Alone-condition clones in our data set. Age did $(p<0.001$ for each clone, Table 3$)$, and brood sex did not ( $p>0.05$ for each clone, Table 3), explain a significant amount of brood size variation in each of the four clones analyzed. This same pattern held

Table 3. Results of ANOVA analyses of brood size variation for each of four individual clones with respect to the factors of maternal age and brood sex

\begin{tabular}{|c|c|c|c|c|c|c|}
\hline Clone & Factor & d.f. & Seq SS & Adj MS & $F$ & $p$ \\
\hline \multirow[t]{4}{*}{ Disp } & Age & 1 & 191.53 & 246.13 & 16.26 & $<0.001$ \\
\hline & BrdSex & 1 & 54.74 & 54.74 & 3.62 & 0.064 \\
\hline & Error & 42 & 635.73 & 15.14 & & \\
\hline & Total & 44 & 882.00 & & & \\
\hline \multirow[t]{4}{*}{ Morgl } & Age & 1 & 3268.1 & 3168.5 & 47.20 & $<0.001$ \\
\hline & BrdSex & 1 & 2.6 & 2.6 & 0.04 & 0.846 \\
\hline & Error & 39 & 2618.3 & 67.1 & & \\
\hline & Total & 41 & 5889.0 & & & \\
\hline \multirow[t]{4}{*}{ War1 } & Age & 1 & 7663.8 & 7396.8 & 127.59 & $<0.001$ \\
\hline & BrdSex & 1 & 79.7 & 79.7 & 1.37 & 0.248 \\
\hline & Error & 39 & 2261.0 & 58.0 & & \\
\hline & Total & 41 & 10004.4 & & & \\
\hline \multirow[t]{4}{*}{ War2 } & Age & 1 & 4266.6 & 3566.2 & 80.27 & $<0.001$ \\
\hline & BrdSex & 1 & 0.5 & 0.5 & 0.01 & 0.913 \\
\hline & Error & 47 & 2088.2 & 44.4 & & \\
\hline & Total & 49 & 6355.4 & & & \\
\hline
\end{tabular}

true when Age was substituted with the reproductive event order (i.e., each brood or ephippium is a reproductive event and ranked sequentially, data not shown). These results suggest the assumption of broods of each sex being the same size, and that there is no significant difference in resource costs for the mother between male and female neonate offspring, is valid.

The GLM evaluating effects on brood size among Alone condition data for the four appropriate clones failed to reveal significant effects of any term involving BrdSex. Thus, all terms involving BrdSex were discarded and a new GLM was evaluated incorporating all six clones. In this GLM, the interaction Age $\times$ Clone explained a significant amount of variation in the number of neonates in each brood $(p<0.001$, Table 2$)$. The terms Age and Clone alone also explained a significant amount of this variation $(p<0.001$ for each, Table 2), but should be interpreted only after their interaction is considered. All GLM-based assumptions of residuals were met. The size of most clones' broods increased with age (Pearson correlation $r=0.484, p<0.001$ ), albeit to different degrees (Fig. 4). Individuals of the Disp clone, however, produced slightly smaller broods with age (Fig. 4a). Age $\times$ Clone remained significant even when Disp was removed from the analysis $\left(\mathrm{F}_{4,202}=6.98, \quad p<0.001\right)$, as did the individual terms Age $\left(\mathrm{F}_{1,202}=357.78, p<0.001\right)$ and Clone $\left(\mathrm{F}_{4,202}=4.54, p=0.002\right)$.

\section{Sex ratio}

The sex ratio of all neonates produced over the length of the experiment varied among clones, and each clone responded differently to crowding. Some clones responded in opposite ways. Morg1 produced a greater proportion of males, while War2 reduced its proportion of males in the Crowded condition (Fig. 5). The interaction of Density $\times$ Clone was responsible for a significant amount of the variation in experiment-long sex ratios $(p<0.001$, Table 2$)$. The term Clone alone also had a significant influence on sex ratios, but as with other analyses the interaction term should take precedence over solitary terms if significant. All GLM-based assumptions of residuals were met.

To evaluate the potential influence of Age on sex ratios, non-consolidated Crowded condition 

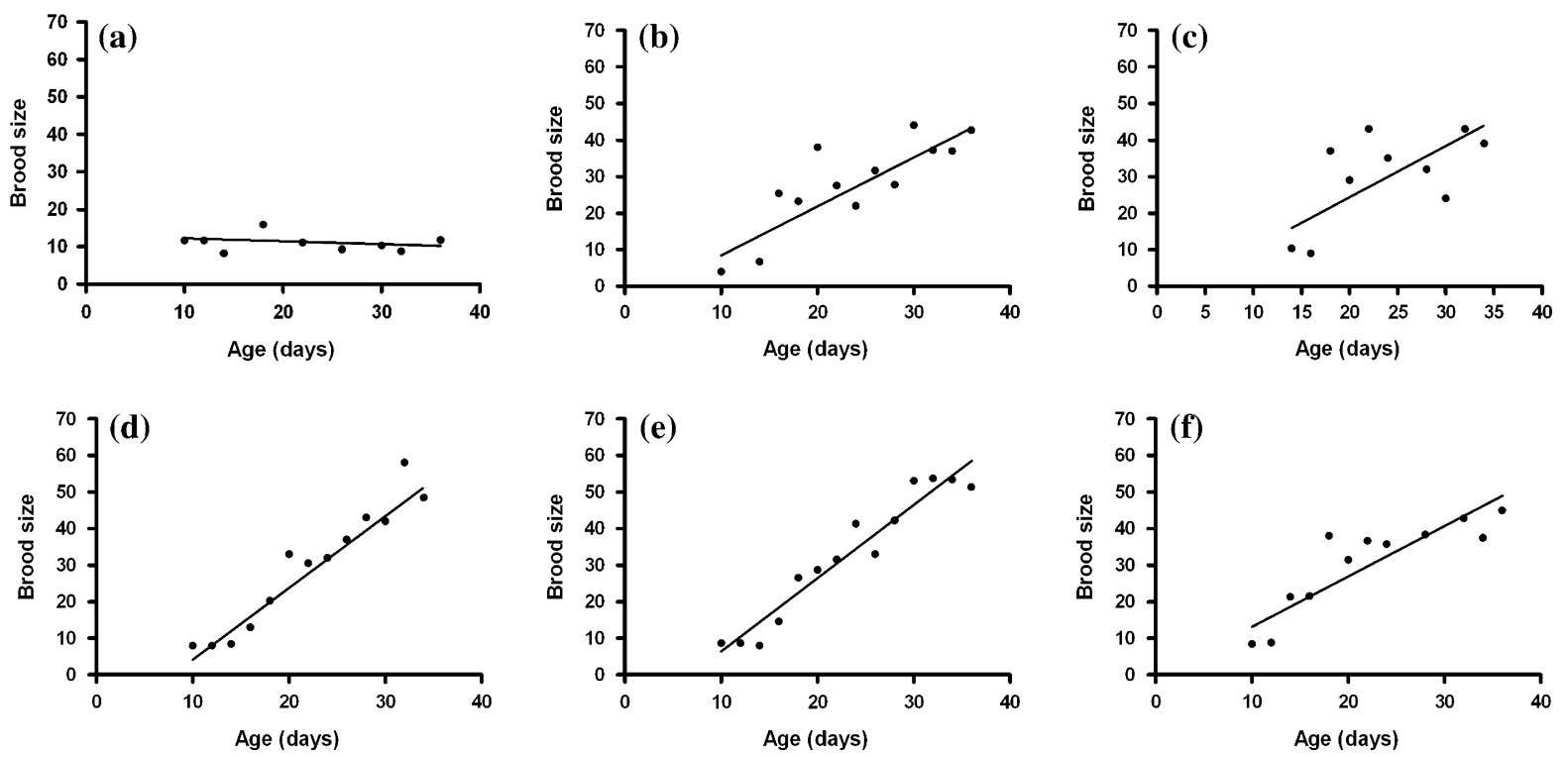

Figure 4. Number of neonates in broods of individuals in the Alone condition, plotted against age of the mother in days. Symbols plot the mean brood size among replicates at a given maternal age. Regression lines across maternal lifetime are shown. Graphs are separated by genotype: (a) Disp, (b) Morg1, (c) Morg2, (d) VBA, (e) War1, and (f) War2.

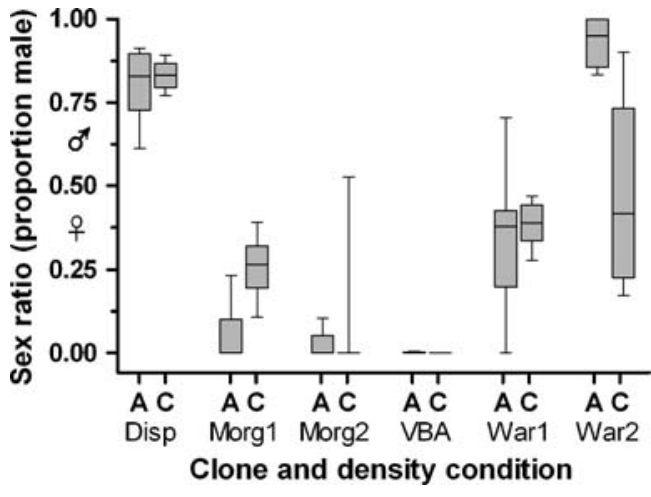

Figure 5. Overall sex ratios (proportion male) of all neonates among clones in the Alone (A) and Crowded (C) conditions. Boxes include the median and span the 25th-75th percentiles. Whiskers span the range of the data.

data were analyzed. Unlike neonate data from the Alone condition, which tended to be all-male or all-female, Crowded condition data were often intermediate due to several daphnids producing broods in the same time frame, often of different sexes. The Age $\times$ Clone interaction explained a significant amount of the variation in sex ratios according to our analyses $\left(\mathrm{F}_{4,286}=2.52, p=0.041\right.$, Fig. 6). Both Age $\left(\mathrm{F}_{1,286}=108.92, p<0.001\right)$ and Clone $\quad\left(\mathrm{F}_{4,286}=7.99, \quad p<0.001\right)$ alone also explained significant variation in sex ratio. All GLM-based assumptions of residuals were met. Each of the five appropriate clones analyzed under Crowded conditions increased their sex ratio in favour of males with age (overall Pearson correlation $r=0.391, p<0.001)$.

To further evaluate the influence of age on sex ratios, we considered whether the age effect was simply due to heavily female-biased first broods. At least one previous study had found no first broods to be male among the three Daphnia clones analyzed (Stross, 1969). Using brood data from the Alone condition we compared sequential broods' sex ratios using $t$-tests. Alpha levels were reduced to $\alpha=0.025$ to reduce risk of type one error with two $t$-tests, as per the Bonferroni correction. We excluded from our analysis all mixed-sex broods and all data from the clones Morg2 and VBA for reasons explained above. Brood data from the remaining four clones were consolidated according to reproductive event, with a replicate's first brood or ephippium given a rank of one, its second reproductive event a two, etc. The mean and standard error sex ratios of broods as the first three reproductive events were $0.194 \pm 0.072$, $0.484 \pm 0.091$ and $0.600 \pm 0.091$, respectively. Broods that were daphnids' first reproductive event 

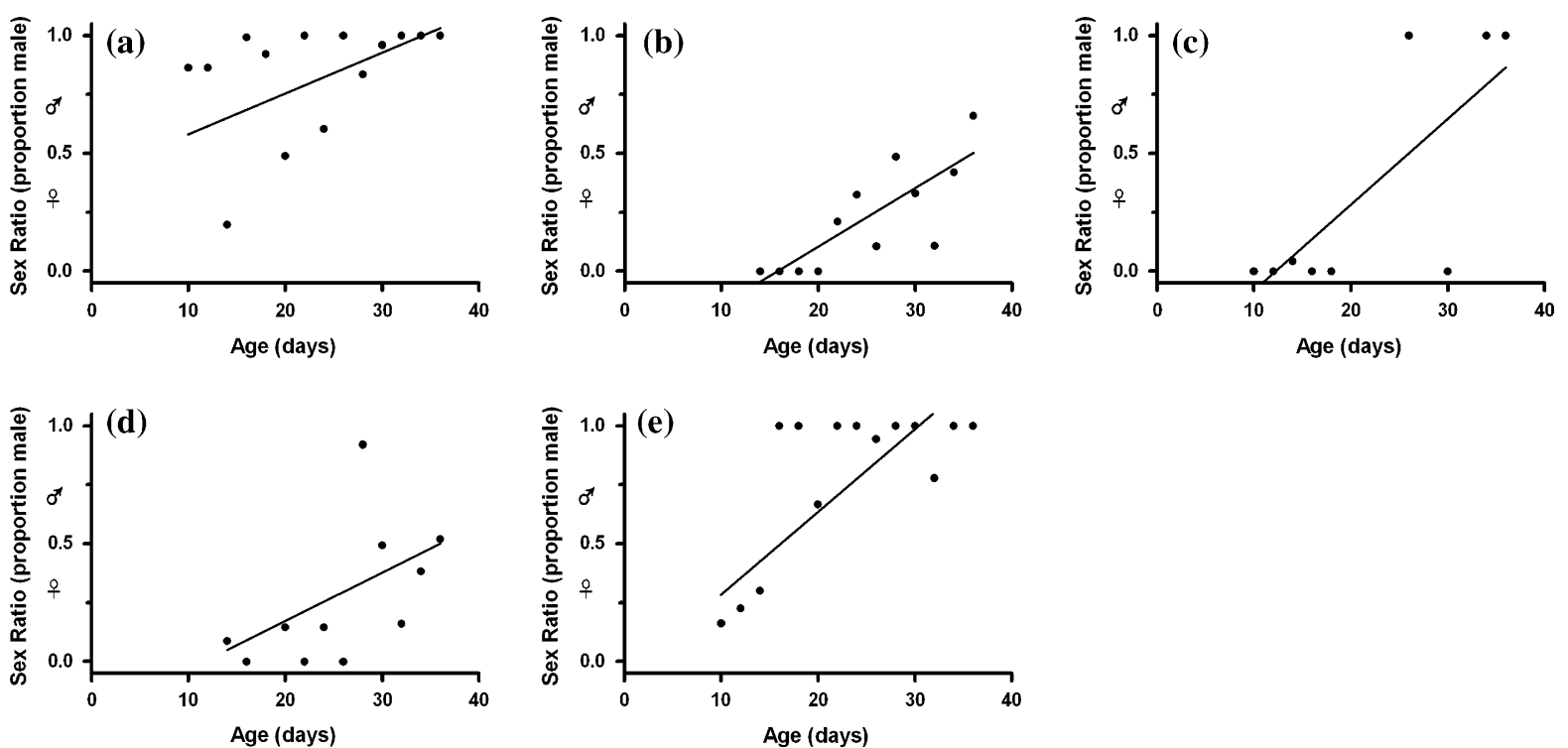

Figure 6. Sex ratio (proportion male) of neonate offspring of five clones in the Crowded condition, in relation to age of mother in days. Symbols plot the mean sex ratios among replicates at a given maternal age. Regression lines across maternal lifetime are shown. Graphs are separated by genotype: (a) Disp, (b) Morg1, (c) Morg2, (d) War1, and (e) War2.

were all-male on several occasions, but were significantly more female-biased than broods that were daphnids' second reproductive event $\left(t_{56}=-2.50, p=0.016\right)$. Each subsequent brood increased its propensity toward male production, but with insignificant differences between broods (sex ratio differences between second and third broods as reproductive events, $t_{58}=-0.90, p=0.371$ ).

\section{Abnormal neonate and mixed-sex brood analyses}

A total of 114 of the 19,755 female neonates collected during this experiment $(0.58 \%)$ were deformed morphologically as described in the Discussion. Only three of these abnormal neonates $(2.63 \%)$ were observed in the Alone condition. Abnormal neonates were produced by each of the experiment's six clones. The assumptions based on residuals necessary for GLMs were violated, preventing its use for analysis of the factors influencing the proportion of female neonates that were deformed. Instead, correlations were analyzed with respect to the fraction of female neonates that were abnormal and the density of adult daphnids. There was a significant positive correlation between the density of adult daphnids and the fraction of female neonates that were abnormal (Pearson correlation $r=0.108, p=0.019)$. Thus as adult density increased, so did the proportion of daughters with developmental abnormalities. Age was significantly and negatively correlated with the fraction of female neonates that were abnormal $(r=-0.115, p=0.012)$, suggesting older daphnids produced relatively fewer abnormal daughters.

Among daphnids in the Alone condition in this experiment, 13 out of 278 broods $(4.68 \%)$ ) contained both males and females. Other studies have found mixed-sex broods to occur at frequencies of $\sim 11 \%$ for D. pulex (Innes, 1997), and 7.6\% (Barker \& Hebert, 1986) and 5.6\% for D. magna (Kleiven et al., 1992) under various conditions.

There has been speculation as to whether or not there is a genetic component to the propensity to produce mixed-sex broods (Innes, 1997). With only 13 mixed-sex broods, the present data set is too small for formal analysis of influential factors. We simply point out that each of the six clones produced at least one mixed-sex brood, with War1 producing the most such broods with six (data not shown).

\section{Discussion}

Crowding provided an important stimulus in reproductive decisions of $D$. pulex. Both the type 
and amount of reproduction varied across density conditions, but remained consistent within each condition within each genotype. This is yet another example of intraclonal phenotypic plasticity in response to environmental stimuli (Lushai et al., 2003; Printes \& Callaghan, 2003). Different genotypes responded to the crowding stimulus in ways that were often similar but sometimes contradictory.

\section{Reproductive responses to crowding}

The per-daphnid rate of ephippia production was significantly higher for crowded daphnids than for those that were alone. In fact, only one of the experiment's six genotypes produced any ephippia in the Alone condition. Genotypes differed in the extent of their ephippia production increase with crowding, but all increased.

Other research has also found ephippia production among $D$. pulex to vary across clones (Innes et al., 2000; Tessier \& Cáceres, 2004) and to increase as a result of crowding (Lürling et al., 2003), sometimes in a clone-specific manner (Berg et al., 2001). Research using other species of Daphnia has yielded similar results for inter-clonal variation in ephippia production among Daphnia pulicaria Forbes (Cáceres \& Tessier, 2004; Tessier \& Cáceres, 2004), and an increase in ephippia production with crowding among clones of D. magna (Ferrari \& Hebert, 1982; Carvalho \& Hughes, 1983), also in a clone-specific manner (Yampolsky, 1992). Some studies have found crowding to only increase ephippia production when combined with additional stimuli, such as reduced food levels (Olmstead \& LeBlanc, 2001) or reduced food levels and low-light photoperiods (Kleiven et al., 1992), but these studies considered only a single clone so their broad applicability may not be valid (see below). Stimuli other than crowding have also been suggested to induce Daphnia ephippia production including: photoperiod (Deng, 1996, 1997b), food level (Deng, 1996), predatory fish (Ślusarczyk, 1995), density of males (Innes, 1997, Innes \& Singleton, 2000), clonal competitive ability (Loaring \& Hebert, 1981), parasite resistance (Mitchell et al., 2004) and the contrast between mother and offspring food levels (LaMontagne \& McCauley, 2001). More multienvironment, multi-genotype research is required to evaluate the effects of interacting stimuli and genotype $\times$ environment interactions on ephippia production.

Just as the effects of crowding on ephippia production were uni-directional but differed in magnitude among clones, crowding reduced perdaphnid experiment-long fecundity in all clones, but to various degrees. This result is supported by previous research on $D$. pulex in which the total number of neonates produced per daphnid was reduced in response to Crowded conditions, but in a clone-specific manner (i.e., significant clone $\mathrm{x}$ density interaction) (Innes \& Singleton, 1994; Innes \& Singleton, 2000; Berg et al., 2001). Microparasites (Lass \& Bittner, 2002), predatory fish (Weber \& Declerck, 1997, Lass \& Bittner, 2002), and temperature (Loaring \& Hebert, 1981) are among the other factors that have been suggested to influence Daphnia fecundity with effects that often vary among clones.

As with other studies, we found a significant negative correlation between the total numbers of ephippia and neonates produced among daphnids in the Crowded condition because a daphnid may only carry an ephippium or a brood at one time (Loaring \& Hebert, 1981; Ruvinsky et al., 1986; Yampolsky, 1992; Berg et al., 2001). This represents an intrinsic trade-off between neonate and resting egg production.

A fraction $(0.58 \%)$ of the female neonates produced in this study were abnormal morphologically. They were characterized by their small size and deformed carapaces and antennae. These developmental abnormalities closely resemble those observed among neonates of D. magna individuals exposed to fenarimol (Mu \& LeBlanc, 2002). Mu \& LeBlanc (2002) further investigated the basis for the developmental abnormalities they observed, and found fenarimol exposure during embryo development to result in reduced ecdysone levels among neonates. We undertook no such biochemical tests of ecdysone levels among daphnids in our experiment. Age was negatively and adult density was positively correlated with the fraction of daughters that were deformed. Further research is required to thoroughly evaluate the embryological reasons for this developmental abnormality and the metabolic and ecological influences on its underlying factors.

The effect of crowding on brood sizes could not be determined in our experiment due to its design. 
Other research has found Daphnia brood sizes to maximize at an intermediate level of crowding (Burns, 1995) and decrease with further crowding (Burns, 1995; Cleuvers et al., 1997; Boersma et al., 1999). Smaller brood sizes in response to crowding may be a result of the smaller size of mothers raised under Crowded conditions (Boersma et al., 1999), or the result of a trade-off between brood size and neonate quality (Mitchell \& Read, 2005). We found brood size, like fecundity, to vary significantly among clones in the Alone condition. As most daphnids grew older, they produced broods with greater numbers of neonates. The magnitude of this age (and size) effect varied across clones (i.e., clone $\times$ age effect), with Disp daphnids notably not increasing their brood sizes with age. Previous research using two clones of $D$. magna found the linear increase of brood size with age to level off in old age (Glazier, 1992). Other research has found that the effect of age upon brood size is one that can be modified by other cues. Upon exposure to a microsporidian parasite, D. magna have been found to increase reproduction at young ages and decrease reproduction when old (Chadwick \& Little, 2005). Daphnia brood size has also been observed to vary in response to food level (Gliwicz \& Guisande, 1992) and exposure to cyanobacterial toxins (Gustafsson et al., 2005).

\section{Offspring sex ratios}

Because offspring sex is determined by non-genetic factors post-fertilization, Daphnia are classified as having environmental sex determination (ESD). Genotypic sex determination (GSD), in contrast, occurs at conception based on genes that are often carried on sex chromosomes (Valenzuela et al., 2003). Although some argue for continued and clear distinctions to be made between ESD and GSD systems (Valenzuela et al., 2003), there is growing support for viewing ESD and GSD as ends of a spectrum between which a continuous range of systems may lie (Sarre et al., 2004). Sex expression of Daphnia has been described as a result of genotype-by-environment interactions (Yampolsky, 1992; Innes, 1997; Innes \& Singleton, 2000), a description our results support.

Fisher's rationale behind selection for a 1:1 operational sex ratio holds true only if sons and daughters cost equal resources to their mothers
(Fisher, 1930; Carvalho et al., 1998). Therefore, the relative costs of male and female neonates to mothers must be considered before D. pulex sex ratios can be evaluated in full. Three previous studies have found no difference in sizes of male and female broods among clones of D. magna (Barker \& Hebert, 1986; Hobæk \& Larsson, 1990) and D. pulex (Innes \& Singleton, 2000). One of these studies used only a single clone, so its results should be treated with caution (Hobæk \& Larsson, 1990). The statistical methods used in the other two studies lacked the power of the one used here because they evaluated each clone separately.

Whether we used within-clone tests or all-clone models, we found no significant difference in the sizes of male and female broods among maleproducing clones in the Alone condition. We therefore conclude that if there are differential costs in male and female neonate production, they are either minute or resources are redistributed from other functions to accommodate the more expensive sex. It is likely that male and female neonates cost the same to a mother, thus sex allocation and sex ratio of neonates can be considered equal.

One clone (VBA) was reluctant to produce males under either density condition, producing only 2 males among 8159 neonates. This was surprising, as numerous males had been observed in stock cultures of this clone prior to this experiment. This provides further evidence that the cues to male production vary across clones.

Among all five male-producing clones in Crowded conditions, there was a significant increase in the sex ratio in favour of males as daphnids grew older. This was true to such an extent that the two FP clones (Disp and War2) were producing male broods almost exclusively by the end of the experiment. Although the effects of seasonal progression on the ratio of males in ponds has been studied (Barker \& Hebert, 1986; Innes, 1997), little research has explicitly examined changes in offspring sex ratio within the lifetime of individual daphnids. Increased male production with age has been observed with a single clone of D. magna (Hobæk \& Larsson, 1990), and another study found no first-broods of three Daphnia clones to be male (Stross, 1969). The single $D$. magna clone used in a study by Olmstead \& LeBlanc (2001) only increased its male production 
with age when under certain environmental conditions and the juvenile hormone analog methoprene was added; otherwise this clone reduced its male production with age.

More research is required into the effects of age upon offspring sex ratios, but we will speculate on potential selective pressures for increased male production with age. These pressures assume sexuality and focus on sexual selection. Sexual selection is of little current relevance to OP lines, but may still be entrenched among OP genomes due to their sexual origins.

We assume males to be more variable in reproductive output than females (Trivers \& Willard, 1973). Little is currently known about sexual selection among Daphnia (Brewer, 1998) other than males can fertilize $>20$ females in one day, and mate with females regardless of genetic similarity (Winsor \& Innes, 2002). Future research on pre- and post-copulatory sexual selection under a variety of conditions would be beneficial to our understanding of Daphnia. Below are three potential evolutionary reasons for increased male production with maternal age.

First, increased male-production with age may be a bet-hedging strategy, whereby males are only produced after it has been ensured that some females have been produced, since males are a riskier investment. It has been demonstrated mathematically that generations of low fitness affect the overall geometric mean of fitness more than those of high fitness (Vrijenhoek, 1998). Thus a 'safe' female-first strategy may be beneficial even if the various low- and high-reproductive generations of an early male production strategy would otherwise seem to cancel each-other out.

Second, assuming males are better able than females to translate high fitness into increased offspring production, if offspring quality increases with maternal age then selection should favour an increased male-bias with maternal age (Trivers \& Willard, 1973). Offspring quality could increase with maternal age simply as a result of older, larger females being able to invest more resources into offspring. Indeed, a study using two genotypes of $D$. magna found females to produce eggs of greater mass with age (Glazier, 1992). In support of this resource-investment-in-males theory, bird offspring sex ratios increase in male-bias with increased maternal condition and/or maternal food intake (Whittingham et al., 2005 and refs therein).

Finally, in addition to being able to allocate increased resources to offspring to increase their quality, an older mother may also indirectly ensure genetic quality for her offspring. A long-lived daphnid is generally of higher quality than those that died at a younger age. Therefore, increased male production among older daphnids may have been selected as a way for high-quality genotypes to produce the offspring sex that can best convert high genetic quality into increased reproductive output.

Offspring sex ratios of Daphnia are often reported to become male-biased when the mother is exposed to either Crowded conditions, or the cues within Daphnia-crowded water (Hobæk \& Larsson, 1990; Kleiven et al., 1992; Innes et al., 2000; Innes \& Singleton, 2000). It is often suggested that increased male production is induced by similar stimuli as ephippia production, just at a more relaxed level (Ferrari \& Hebert, 1982, Kleiven et al., 1992). This is supported by Daphnia field sample studies finding high levels of males in a pond or lake 2 weeks before high levels of ephippial females (De Meester \& Vanoverbeke, 1999; Cáceres $\&$ Tessier, 2004; Spaak et al., 2004). In addition to crowding, stimuli reported to alter Daphnia offspring sex ratios include photoperiod (Ferrari \& Hebert, 1982, Korpelainen, 1986, Innes et al., 2000, Zhang \& Baer, 2000), temperature (Korpelainen, 1986), food level (Ferrari \& Hebert, 1982; Zhang \& Baer, 2000), and exposure to predatory fish (Boersma et al., 1998).

In contrast to some of the literature cited above, our results do not support a simple relationship between increased crowding and increased male-bias among $D$. pulex sex ratios. We found significant differences not only among clones' sex ratios, but also among clones' sex ratio responses to increased density. The clones Morg1 and Warl increased their sex ratio in favour of males under Crowded conditions, while War2 behaved in the opposite way by producing fewer males under Crowded conditions. Disp, meanwhile, did not alter its sex ratio much either way with crowding.

How can our findings of inter-clonal variation in sex ratio responses to density be reconciled with the above-cited literature describing a simple 
positive relationship between crowding and sex ratios? The experimental design of some studies prevented detailed quantification of offspring sex ratios in response to crowding (Innes \& Singleton, 2000), or only studied the effects of crowding when combined with photoperiod and thus could not tease apart the effects of the two cues (Innes et al., 2000). Two studies used the same single genotype of D. magna and thus could not detect inter-clonal variation (Hobæk \& Larsson, 1990; Kleiven et al., 1992).

Studies that have tested for inter-clonal variation in sex ratios have found it, among clones of $D$. pulex (Innes \& Dunbrack, 1993; Innes, 1997; Innes et al., 2000; Innes \& Singleton, 2000), clones of $D$. pulicaria (Tessier \& Cáceres, 2004), and both clones (Korpelainen, 1986) and populations (Ferrari \& Hebert, 1982) of D. magna. In fact, the significant clone $\times$ density interaction affecting sex ratios in the present study was also found to significantly influence sex ratios in other studies on $D$. pulex (Ruvinsky et al., 1986; Innes \& Singleton, 1994) and D. magna (Yampolsky, 1992). A population $\times$ density interaction similarly influenced sex ratios in a component of a study on $D$. pulex that did not distinguish between clones (Berg et al., 2001). We are also not alone in reporting crowding to induce different clones to respond with sex ratios biased in opposite directions (Innes \& Singleton, 1994).

Applicability of results from the laboratory to nature is difficult to assess. However, other researchers have found their lab results to match well with field results for D. pulex (Innes, 1997; Tessier \& Cáceres, 2004), providing reason to believe the same may be true for the present experiment.

\section{Male production among asexual D. pulex}

Previous research suggests male production among OP genotypes is much-reduced compared to male production among FP genotypes (Innes et al., 2000). Any male production at all among OP clones seems paradoxical, but may in fact be an adaptive trait. Males from OP clones can mate with FP females to create novel OP lines (Innes \& Hebert, 1988). There is evidence that obligate parthenogenesis has been spreading across North American D. pulex populations in a contagious fashion through such FP-OP matings (Paland et al., 2005). These new OP lines are expected to benefit from the half-genome contribution of the FP mother, as it is expected to be both locally adapted and less mutated than that of the OP father (Simon et al., 2003). Novel asexual lineages are believed to have resulted from similar matings between asexual-lineage males and sexual-lineage females in other species (Weinzierl et al., 1999, Simon et al., 2002).

Although selection may favour male production among OP clones in areas near FP populations of D. pulex (Innes et al., 2000), why would male production continue among OP clones far away from FP populations? Asexual genomes by their very definition do not recombine with others, and thus change very little over generations. Selection at each locus is reduced in an asexual genome (Rice \& Chippindale, 2001; Betancourt \& Presgraves, 2002; Hadany \& Feldmans, 2005), so traits may be retained after they have ceased to be adaptive. Put simply, an asexual genome has trouble separating itself from its maker. This applies to sexual selection strategies (see above), male production, and other traits adaptive to sexuality.

It would be interesting to contrast levels of male production from OP populations at the FPOP overlap range of middle North America with those from the Northeast of the continent whence obligate parthenogenesis is believed to have originated, and where obligate parthenogenesis is now ubiquitous among D. pulex populations (Paland et al., 2005). Is selection against male production in the Northeast strong enough to have eliminated those genotypes with heavy male production and hence reduce genotypic diversity? Or, is it weak enough that its effects have been largely overridden by the overall fitness of the rest of the genome, and from the benefits individual genotypes may enjoy in populations with low genetic diversity? Has sufficient time passed for selection against male production to have had an effect? It is also possible that males serve a yet-unknown selected function, and their continued production is in fact adaptive.

\section{Daphnia pulex crowding infochemicals}

Having discussed the effects of crowding on $D$. pulex, let us now consider the very nature of 
this crowding stimulus. What are the infochemicals used by Daphnia to detect crowding, and how specific are they? Although physical interaction with other daphnids may increase the stimulus, the mere water in which daphnids have lived is sufficient to induce a crowding response among Daphnia (Larsson, 1991). Six kairomones have recently been isolated from $D$. pulex that induce morphological changes in algal prey (Yasumoto et al., 2005). Whether these kairomones are the crowding infochemicals detected by fellow daphnids remains to be seen, but their synthesis allows future research to test the possibility.

The specificity of Daphnia infochemicals is a matter of evolutionary importance. If daphnids were able to distinguish between infochemicals emitted by males and females then they could conceivably adjust their offspring sex ratios or reproductive behaviour accordingly, as some monoecious plants do depending on the amount of pollen they detect (López \& Domínguez, 2003). The population demographics experienced by a mother, however, may not reliably predict those her offspring will experience at sexual maturity, which may be a reason why some bird species, for instance, do not employ this strategy (Bensch et al., 1999). A study using a single genotype of $D$. magna failed to reveal any distinction between male and female cues in swarming behaviour (Crease \& Hebert, 1983).

If genotypes of Daphnia were able to distinguish between their own infochemicals and those of others in the same species, then they would be better able to determine outbreeding opportunities. Within-clone matings are known to produce offspring with fitness that is variable but reduced on average (Innes, 1989; Innes \& Dunbrack, 1993; Deng, 1997a; Salathé \& Ebert, 2003). Perhaps $D$. pulex adjusts its reproduction in response to outbreeding opportunities, just as female ambrosia beetles adjust their offspring sex ratio depending on outbreeding opportunities (Peer \& Taborsky, 2004).

Are infochemicals detected across species within the genus Daphnia, or even within the family Cladocera? Inter-species detection of infochemicals decreases their reliability of prediction, and may select for the utilization of alternative predictors of environmental conditions (Serra et al., 2005). As with Daphnia, crowding induces a sexual response in many rotifers (Carmona et al., 1993). However, both experimental and theoretical evidence suggests some rotifers use the progression of generations as a sex-inducing cue instead of crowding, likely because crowding cues are not species-specific and hence lack accuracy (Serra et al., 2005). What little research conducted on inter-species detection of Daphnia infochemicals has used single genotypes, so inter-species response levels could not be compared to intraspecific intergenotype response levels (Hobæk \& Larsson, 1990; Burns, 1995; Lürling et al., 2003).

Many aspects of Daphnia infochemicals have implications that extend to experimental design. Much research has been conducted on the effects of fish-inhabited water on Daphnia, with effects attributed to fish kairomones (e.g., Boersma et al., 1998; Weetman \& Atkinson, 2002). However, fish were almost always fed Daphnia, and evidence suggests it is not fish kairomones but latent daphnia alarm cues activated by bacteria and/or enzymes in fish digestive tracts to which daphnids respond (Stabell et al., 2003). A similar mechanism may be responsible for observed daphnid responses to water exposed to invertebrate predators (e.g., Pijanowska \& Kowalczewski, 1997). And in a study on the effects of crowding upon $D$. pulex reproduction, water used for all clones came from one pond (Berg et al., 2001): a not-uncommon method among Daphnia laboratories (e.g., Deng, 1997b). The authors point out that this may have differentially affected the clone originating from this pond compared to the other clones in the experiment, but rate this an unlikely possibility (Berg et al., 2001).

\section{Research implications and directions}

This experiment and others like it have provided ample evidence for different genotypes of Daphnia responding differently to stimuli. A great deal of published research ignores inter-genotype variation, drawing species-wide conclusions from a single genotype of Daphnia (e.g., Kleiven et al., 1992; Alekseev \& Lampert, 2001; LaMontagne \& McCauley, 2001; Kessler \& Lampert, 2004; Mikulski et al., 2005). Drawing species-wide conclusions from multiple replicates of the same genotype can be considered a form of pseudoreplication, and should be avoided (Hurlbert, 1984). 
Testing regulations of freshwater effluent established by governments worldwide require no more than one genotype of Daphnia to be used (OECD, 1998; Environment Canada, 2000; USEPA, 2002). In fact, some researchers and regulations have advocated the widespread use of a single specific genotype to aid repeatability between laboratories (Baird et al., 1991; OECD, 1998).

By allowing tests to be conducted on a single genotype, government regulations are making an improper assumption of low inter-genotype variation. If repeatability is the justification for these regulations, then universal usage of a specific battery of genotypes would improve upon current regulations' repeatability. If labour costs to company laboratories for using several instead of single genotypes is the rationale behind current regulations, then we must re-examine the value we place on the well-being of our aquatic environment (De Meester \& Declerck, 2005).

\section{Conclusions}

Crowding is an important stimulus to reproductive decisions of Daphnia pulex. Six genotypes of $D$. pulex were raised under two different density regimes and reproductive output was observed. Four of these genotypes reproduce via obligate parthenogenesis and two by facultative parthenogenesis. Insufficient sample sizes prevented prudent comparison of reproductive output between the two reproductive types.

Crowding induced increased ephippia production and decreased neonate production among all genotypes. Neonate brood sizes increased with maternal age for most genotypes, and offspring sex ratios grew more male-biased with maternal age for all relevant genotypes. Most daphnids modified offspring sex ratios in response to crowding, but in genotype-specific directions that were often contradictory. The genotype $\times$ environment interaction for sex ratios was the most obvious of several in this investigation. Inter-genotypic variation in response to environmental stimuli highlight the importance of using more than one genotype in Daphnia research.

Several potential evolutionary explanations are explored for increased male production with age, male production among asexual Daphnia and a potential environment-induced trade-off between offspring quantity and quality. We also discuss the nature of Daphnia crowding infochemicals, and comment on abnormal neonate development.

The crowding stimulus is an important one in D. pulex reproductive decisions, but one to which different genotypes may respond in different ways.

\section{Acknowledgements}

We thank Murray Colbo, France Dufresne, Doreen Singleton, Brian Staveley, and two anonymous reviewers for helpful comments on previous versions of the manuscript. Funding for this work was provided by a Natural Sciences and Engineering Research Council, Canada grant to D. J. I. and a Memorial University graduate fellowship to J. M. F.

\section{References}

Alekseev, V. \& W. Lampert, 2001. Maternal control of restingegg production in Daphnia. Nature 414: 899-901.

Antunes, S. C., B. B. Castro \& F. Gonçalves, 2003. Chronic responses of different clones of Daphnia longispina (field and ephippia) to different food levels. Acta Oecologica 24: S325S332.

Baird, D. J., I. Barber, M. Bradley, A. M. V. M. Soares \& P. Calow, 1991. A comparative study of genotype sensitivity to acute toxic stress using clones of Daphnia magna Straus. Ecotoxicology and Environmental Safety 21: 257-265.

Banta, A. M. \& L. A. Brown, 1929. Control of sex in Cladocera. I. crowding the mothers as a means of controlling male production. Physiological Zoölogy 2: 80-92.

Barker, D. M. \& P. D. N. Hebert, 1986. Secondary sex ratio of the cyclic parthenogen Daphnia magna (Crustacea: Cladocera) in the Canadian Arctic. Canadian Journal of Zoology 64: 1137-1143.

Bensch, S., H. Westerdahl, B. Hansson \& D. Hasselquist, 1999. Do females adjust the sex of their offspring in relation to the breeding sex ratio?. Journal of Evolutionary Biology 12: 1104-1109.

Berg, L. M., S. Pálsson \& M. Lascoux, 2001. Fitness and sexual response to population density in Daphnia pulex. Freshwater Biology 46: 667-677.

Betancourt, A. J. \& D. C. Presgraves, 2002. Linkage limits the power of natural selection in Drosophila. Proceedings of the National Academy of Sciences USA 99: 13616-13620.

Boersma, M., L. De Meester \& P. Spaak, 1999. Environmental stress and local adaptation in Daphnia magna. Limnology and Oceanography 44: 393-402. 
Boersma, M., P. Spaak \& L. De Meester, 1998. Predatormediated plasticity in morphology, life history, and behavior of Daphnia: the uncoupling of responses. American Naturalist 152: 237-248

Brewer, M. C., 1998. Mating behaviours of Daphnia pulicaria, a cyclic parthenogen: comparisons with copepods. Philosophical Transactions of the Royal Society of London B 353: 805-815.

Burns, C. W., 1995. Effects of crowding and different food levels on growth and reproductive investment of Daphnia. Oecologia 101: 234-244.

Cáceres, C. E. \& A. J. Tessier, 2004. Incidence of diapause varies among populations of Daphnia pulicaria. Oecologia 141: 425-431

Carmona, M. J., M. Serra \& M. R. Miracle, 1993. Relationships between mixis in Brachionus plicatilis and preconditioning of culture medium by crowding. Hydrobiologia 255/ 256: $145-152$.

Carvalho, A. B., M. C. Sampaio, F. R. Varandas \& L. B. Klaczko, 1998. An experimental demonstration of Fisher's Principle: evolution of sexual proportion by natural selection. Genetics 148: 719-731.

Carvalho, G. R. \& R. N. Hughes, 1983. The effect of food availability, female culture-density and photoperiod on ephippia production in Daphnia magna Straus (Crustacea: Cladocera). Freshwater Biology 13: 37-46.

Chadwick, W. \& T. J. Little, 2005. A parasite-mediated lifehistory shift in Daphnia magna. Proceedings of the Royal Society of London B 272: 505-509.

Cleuvers, M., B. Goser \& H.-T. Ratte, 1997. Life-history shift by intraspecific interaction in Daphnia magna: change in reproduction from quantity to quality. Oecologia 110: 337-345.

Crease, T. J. \& P. D. N. Hebert, 1983. A test for the production of sexual pheromones by Daphnia magna (Crustacea: Cladocera). Freshwater Biology 13: 491-496.

De Meester, L. \& S. Declerck, 2005. The study of biodiversity in freshwater habitats: societal relevance and suggestions for priorities in science policy. Hydrobiologia 542: 1-9.

De Meester, L. \& J. Vanoverbeke, 1999. An uncoupling of male and sexual egg production leads to reduced inbreeding in the cyclical parthenogen Daphnia. Proceedings of the Royal Society of London B 266: 2471-2477.

Deng, H.-W., 1996. Environmental and genetic control of sexual reproduction in Daphnia. Heredity 76: 449-458.

Deng, H.-W., 1997a. Increase in developmental instability upon inbreeding in Daphnia. Heredity 78: 182-189.

Deng, H.-W., 1997b. Photoperiodic response of sexual reproduction in the Daphnia pulex group is reversed in two distinct habitats. Limnology and Oceanography 42: 609-611.

Dudycha, J. L., 2004. Mortality dynamics of Daphnia in contrasting habitats and their role in ecological divergence. Freshwater Biology 49: 505-514

Environment Canada, 2000. Biological Test Method: Reference Method for Determining Acute Lethality of Effluents to Daphnia magna. Environmental Technology Centre, Ottawa.

Ferrari, D. C. \& P. D. N. Hebert, 1982. The induction of sexual reproduction in Daphnia magna: genetic differences between arctic and temperate populations. Canadian Journal of Zoology 60: 2143-2148.
Fisher, R. A., 1930. The Genetical Theory of Natural Selection. Claredon Press, Oxford.

Fitzsimmons, J. M. \& D. J. Innes, 2005. No evidence of $\mathrm{Wol}$ bachia among Great Lakes area populations of Daphnia pulex (Crustacea: Cladocera). Journal of Plankton Research 27: 121-124.

Glazier, D. S., 1992. Effects of food, genotype, and maternal size and age on offspring investment in Daphnia magna. Ecology 73: 910-926.

Gliwicz, Z. M. \& C. Guisande, 1992. Family planning in Daphnia: resistance to starvation in offspring born to mothers grown at different food levels. Oecologia 91: 463467.

Gustafsson, S., K. Rengefors \& L.-A. Hansson, 2005. Increased consumer fitness following transfer of toxin tolerance to offspring via maternal effects. Ecology 86: 2561-2567.

Hadany, L. \& M. W. Feldman, 2005. Evolutionary traction: the cost of adaptation and the evolution of sex. Journal of Evolutionary Biology 18: 309-314.

Hebert, P. D. N., 1987. Genotypic characteristics of the Cladocera. Hydrobiologia 145: 183-193.

Hebert, P. D. N., M. J. Beaton, S. S. Schwartz \& D. J. Stanton, 1989. Polyphyletic origins of asexuality in Daphnia pulex. I. breeding-system variation and levels of clonal diversity. Evolution 43: 1004-1015.

Hebert, P. D. N. \& T. Crease, 1983. Clonal diversity in populations of Daphnia pulex reproducing by obligate parthenogenesis. Heredity 51: 353-369.

Hobæk, A. \& P. Larsson, 1990. Sex determination in Daphnia magna. Ecology 71: 2255-2268.

Hurlbert, S. H., 1984. Pseudoreplication and the design of ecological field experiments. Ecological Monographs 54: 187-211.

Innes, D. J., 1989. Genetics of Daphnia obtusa: genetic load and linkage analysis in a cyclical parthenogen. Journal of Heredity 80: 6-10.

Innes, D. J., 1997. Sexual reproduction of Daphnia pulex in a temporary habitat. Oecologia 111: 53-60.

Innes, D. J. \& R. L. Dunbrack, 1993. Sex allocation variation in Daphnia pulex. Journal of Evolutionary Biology 6: 559-575.

Innes, D. J., C. J. Fox \& G. L. Winsor, 2000. Avoiding the cost of males in obligately asexual Daphnia pulex (Leydig). Proceedings of the Royal Society of London B 267: 991-997.

Innes, D. J. \& P. D. N. Hebert, 1988. The origin and genetic basis of obligate parthenogenesis in Daphnia pulex. Evolution 42: 1024-1035.

Innes, D. J., S. S. Schwartz \& P. D. N. Hebert, 1986. Genotypic diversity and variation in mode of reproduction among populations in the Daphnia pulex group. Heredity 57: 345-355.

Innes, D. J. \& D. R. Singleton, 1994. Variation in reproduction and sex allocation among clones of Daphnia pulex. In Beaumont, A. R. (ed.), Genetics and Evolution of Aquatic Organisms. Chapman and Hall, London: 335-342.

Innes, D. J. \& D. R. Singleton, 2000. Variation in allocation to sexual and asexual reproduction among clones of cyclically parthenogenetic Daphnia pulex (Crustacea: Cladocera). Biological Journal of the Linnean Society 71: 771-787. 
Kerfoot, W. C. \& L. J. Weider, 2004. Experimental paleoecology (resurrection ecology): chasing Van Valen's Red Queen hypothesis. Limnology and Oceanography 49: 1300-1316.

Kessler, K. \& W. Lampert, 2004. Fitness optimization of Daphnia in a trade-off between food and temperature. Oecologia 140: 381-387.

Kleiven, O. T., P. Larsson \& A. Hobæk, 1992. Sexual reproduction in Daphnia magna requires three stimuli. Oikos 65: 197-206.

Korpelainen, H., 1986. The effects of temperature and photoperiod on life history parameters of Daphnia magna (Crustacea: Cladocera). Freshwater Biology 16: 615-620.

Korpelainen, H., 1992. Lowered female reproductive effort as an indicator for increased male production and sexuality in Daphnia (Crustacea: Cladocera). Invertebrate Reproduction and Development 22: 281-290.

LaMontagne, J. M. \& E. McCauley, 2001. Maternal effects in Daphnia: what mothers are telling their offspring and do they listen? Ecology Letters 4: 64-71.

Larsson, P., 1991. Intraspecific variability in response to stimuli for male and ephippia formation in Daphnia pulex. Hydrobiologia 225: 281-290.

Lass, S. \& K. Bittner, 2002. Facing multiple enemies: parasitised hosts respond to predator kairomones. Oecologia 132: 344-349.

Loaring, J. M. \& P. D. N. Hebert, 1981. Ecological differences among clones of Daphnia pulex Leydig. Oecologia 51: 162168.

López, S. \& C. A. Domínguez, 2003. Sex choice in plants: facultative adjustment of the sex ratio in the perennial herb Begonia gracilis. Journal of Evolutionary Biology 16: 1177-1185.

Lürling, M., F. Roozen, E. Van Donk \& B. Goser, 2003. Response of Daphnia to substances released from crowded congeners and conspecifics. Journal of Plankton Research 25: 967-978.

Lushai, G., H. D. Loxdale \& J. A. Allen, 2003. The dynamic clonal genome and its adaptive potential. Biological Journal of the Linnean Society 79: 193-208.

Lynch, M., K. Spitze \& T. Crease, 1989. The distribution of lifehistory variation in the Daphnia pulex complex. Evolution 43: $1724-1736$.

Lynch, M., L. J. Weider \& W. Lampert, 1986. Measurement of the carbon balance in Daphnia. Limnology and Oceanography 31: 17-33.

Mikulski, A., M. Czernik \& J. Pijanowska, 2005. Induction time and reversibility of changes in Daphnia life history caused by the presence of fish. Journal of Plankton Research 27: 757-762.

Mitchell, S. E. \& A. F. Read, 2005. Poor maternal environment enhances offspring disease resistance in an invertebrate. Proceedings of the Royal Society of London B 272: 26012607.

Mitchell, S. E., A. F. Read \& T. J. Little, 2004. The effect of a pathogen epidemic on the genetic structure and reproductive strategy of the crustacean Daphnia magna. Ecology Letters 7: 848-858.

Mitchell, S. E., E. S. Rogers, T. J. Little \& A. F. Read, 2005. Host-parasite and genotype-by-environment interactions: temperature modifies potential for selection by a sterilizing pathogen. Evolution 59: 70-80.

Mu, X. \& G. A. LeBlanc, 2002. Environmental antiecdysteroids alter embryo development in the crustacean Daphnia magna. Journal of Experimental Zoology 292: 287-292.

Nelson, W. A., E. McCauley \& F. J. Wrona, 2005. Stagestructured cycles promote genetic diversity in a predatorprey system of Daphnia and algae. Nature 433: 413-417.

OECD, 1998. OECD Guidelines for Testing of Chemicals: Daphnia magna Reproduction Test. Organisation for Economic Co-operation and Development, Paris.

Olmstead, A. W. \& G. A. LeBlanc, 2001. Temporal and quantitative changes in sexual reproductive cycling of the cladoceran Daphnia magna by a juvenile hormone analog. Journal of Experimental Zoology 290: 148-155.

Olmstead, A. W. \& G. A. LeBlanc, 2002. Juvenoid hormone methyl farnesoate is a sex determinant in the crustacean Daphnia magna. Journal of Experimental Zoology 293: 736-739.

Paland, S., J. K. Colbourne \& M. Lynch, 2005. Evolutionary history of contagious asexuality in Daphnia pulex. Evolution 59: 800-813.

Peer, K. \& M. Taborsky, 2004. Female ambrosia beetles adjust their offspring sex ratio according to outbreeding opportunities for their sons. Journal of Evolutionary Biology 17: 257-264.

Pijanowska, J. \& A. Kowalczewski, 1997. Cues from injured Daphnia and from cyclopoids feeding on Daphnia can modify life histories of conspecifics. Hydrobiologia 350: 99-103.

Printes, L. B. \& A. Callaghan, 2003. Intraclonal variability in Daphnia acetylcholinesterase activity: the implications for its applicability as a biomarker. Environmental Toxicology and Chemistry 22: 2042-2047.

Rice, W. R. \& A. K. Chippindale, 2001. Sexual recombination and the power of selection. Science 294: 555-559.

Ruvinsky, A. O., A. A. Perelygin, Y. I. Lobkov \& D. K. Belyaev, 1986. Factors organising and maintaining polymorphism in a cyclic parthenogenetic species: Daphnia pulex. Heredity 57: 15-22.

Salathé, P. \& D. Ebert, 2003. The effects of parasitism and inbreeding on the competitive ability in Daphnia magna: evidence for synergistic epistasis. Journal of Evolutionary Biology 16: 976-985.

Sanders, R. W., C. E. Williamson, P. L. Stutzman, R. E. Moeller, C. E. Goulden \& R. Aoki-Goldsmith, 1996. Reproductive success of "herbivorous" zooplankton fed algal and nonalgal food resources. Limnology and Oceanography 41: 1295-1305.

Sarre, S. D., A. Georges \& A. Quinn, 2004. The ends of a continuum: genetic and temperature-dependent sex determination in reptiles. BioEssays 26: 639-645.

Serra, M., T. W. Snell \& J. J. Gilbert, 2005. Delayed mixis in rotifers: an adaptive response to the effects of densitydependent sex on population growth. Journal of Plankton Research 27: 37-45.

Simon, J.-C., F. Delmotte, C. Rispe \& T. Crease, 2003. Phylogenetic relationships between parthenogens and their sexual relatives: the possible routes to parthenogenesis in animals. Biological Journal of the Linnean Society 79: 151-163. 
Simon, J.-C., C. Rispe \& P. Sunnucks, 2002. Ecology and evolution of sex in aphids. Trends in Ecology and Evolution 17: 34-39.

Ślusarczyk, M., 1995. Predator-induced diapause in Daphnia. Ecology 76: 1008-1013.

Spaak, P., A. Denk, M. Boersma \& L. J. Weider, 2004. Spatial and temporal patterns of sexual reproduction in a hybrid Daphnia species complex. Journal of Plankton Research 26: 625-635.

Stabell, O. B., F. Ogbebo \& R. Primicerio, 2003. Inducible defences in Daphnia depend on latent alarm signals from conspecific prey activated in predators. Chemical Senses 28: $141-153$.

Stross, R. G., 1969. Photoperiod control of diapause in Daphnia. III. Two-stimulus control of long-day, short-day induction. Biological Bulletin 137: 359-374.

Tessier, A. J. \& C. E. Cáceres, 2004. Differentiation in sex investment by clones and populations of Daphnia. Ecology Letters 7: 695-703.

Trivers, R. L. \& D. E. Willard, 1973. Natural selection of parental ability to vary the sex ratio of offspring. Science 179: 90-92.

USEPA, 2002. Methods for Measuring the Acute Toxicity of Effluents and Receiving Waters to Freshwater and Marine Organisms. U.S. Environmental Protection Agency, Washington.

Valenzuela, N., D. C. Adams \& F. J. Janzen, 2003. Pattern does not equal process: exactly when is sex environmentally determined?. American Naturalist 161: 676-683.

Vrijenhoek, R. C., 1998. Animal clones and diversity: are natural clones generalists or specialists?. BioScience 48: 617-628.
Weber, A. \& S. Declerck, 1997. Phenotypic plasticity of Daphnia life history traits in response to predator kairomones: genetic variability and evolutionary potential. Hydrobiologia 360: 89-99.

Weetman, D. \& D. Atkinson, 2002. Antipredator reaction norms for life history traits in Daphnia pulex: dependence on temperature and food. Oikos 98: 299-307.

Weinzierl, R. P., P. Schmidt \& N. K. Michiels, 1999. High fecundity and low fertility in parthenogenetic planarians. Invertebrate Biology 118: 87-94.

Whittingham, L. A., P. O. Dunn \& J. K. Nooker, 2005. Maternal influences on brood sex ratios: an experimental study in tree swallows. Proceedings of the Royal Society of London B 272: 1775-1780.

Winsor, G. L. \& D. J. Innes, 2002. Sexual reproduction in Daphnia pulex (Crustacea: Cladocera): observations on male mating behaviour and avoidance of inbreeding. Freshwater Biology 47: 441-450.

Yampolsky, L. Y., 1992. Genetic variation in the sexual reproduction rate within a population of a cyclic parthenogen, Daphnia magna. Evolution 46: 833-837.

Yasumoto, K., A. Nishigami, M. Yasumoto, F. Kasai, Y. Okada, T. Kusumi \& T. Ooi, 2005. Aliphatic sulfates released from Daphnia induce morphological defense of phytoplankton: isolation and synthesis of kairomones. Tetrahedron Letters 46: 4765-4767.

Zhang, L. \& K. N. Baer, 2000. The influence of feeding, photoperiod and selected solvents on the reproductive strategies of the water flea, Daphnia magna. Environmental Pollution 110: 425-430. 